\title{
ANÁLISE DAS REDES SOCIAIS DE UMA FÁBRICA DE EQUIPAMENTOS MÉDICOS
}

\section{ANÁLISIS DE LAS REDES SOCIALES DE UNA FÁBRICA DE EQUIPOS MÉDICOS}

\section{ANALYSIS OF THE SOCIAL NETWORKS OF A MEDICAL EQUIPMENT FACTORY}

\author{
${ }^{1}$ Gislaine Cristina dos Santos Teixeira \\ ${ }^{2}$ Cíntia Cristina Silva de Araújo
}

\section{RESUMO}

As redes sociais representam um conjunto de participantes autônomos unidos por ideias e recursos em torno de interesses compartilhados. A análise das redes sociais é uma técnica usada para entender os fluxos de informação e as construções sociais das interações humanas. Apesar das redes sociais estarem presentes no ambiente corporativo, sua influência na condução dos negócios não é levada em conta no alinhamento estratégico das organizações. Neste contexto, o objetivo desta pesquisa foi analisar os contatos desenvolvidos pelos funcionários de uma fábrica brasileira de equipamentos médicos, visando entender suas interações e a influência destas interações na estrutura organizacional. Para cumprir este objetivo, utilizamos a técnica de análise de redes sociais de blockmodels. Este relato descreve os resultados de nosso estudo aplicado, com abordagem qualitativa em que foi adotada a metodologia de pesquisa diagnóstica. A análise das matrizes nos permitiu identificar os centros e periferias das redes de contatos. Dentre os diagnósticos apresentados estão a necessidade de estruturação de processos de comunicação para diminuir os problemas de falhas de comunicação e diminuir o isolamento de alguns departamentos.

Palavras-chave: Redes sociais; Estrutura Organizacional; Estudo Organizacional

\section{RESUMEN}

Las redes sociales integran a un conjunto de participantes autónomos unidos por ideas y recursos en torno a intereses compartidos. El análisis de las redes sociales es una técnica utilizada para entender los flujos de información y los constructos sociales de aquellas

\footnotetext{
${ }^{1}$ Doctorate Student at Nove de Julho University - UNINOVE, São Paulo (Brasil). gislainesteixeira@gmail.com

${ }^{2}$ Doctorate Student at Nove de Julho University - UNINOVE, São Paulo (Brasil). cintyaraujo@gmail.com
} 
Análise das Redes Sociais de uma Fábrica de Equipamentos Médicos

interacciones humanas. A pesar de la presencia de las redes sociales en el ambiente corporativo, su influencia en la conducción de negocios no es considerada como parte del alineamiento estratégico de las organizaciones. En este contexto, el objetivo de este estudio fue analizar los contactos desarrollados por los funcionarios de una fábrica brasileña de equipamiento médico, buscando entender sus interacciones y la influencia de estas en la estructura organizacional. Para cumplir este objetivo, utilizamos la técnica de análisis de redes sociales de blockmodels. Este informe describe los resultados de nuestro estudio, aplicado con un abordaje cualitativo con el que fue adoptada la metodología de investigación diagnóstica. El análisis de las matrices nos permitió identificar las áreas centrales y periféricas de las redes de contactos. Dentro de los diagnósticos presentados están las necesidades de estructuración de procesos comunicacionales para diminuir los problemas de fallas de comunicación y aminorar el aislamiento de algunos departamentos.

Palabra llave: Redes sociales; estrutura organizacional; estudo organizacional

\begin{abstract}
Social networks represent a set of independent participants that are connected through ideas and resources due to interests in common (Marteleto, 2001).The analysis of social networks is a technique used to understand the information flows and the social constructs in the human interactions. Even though social netweks are well presents in corporate settings, their influence in business is not taken into account in the stratetigc alignment of organizations. In this context, the objective of this research is to analyze the social contacts among employees of a Brazilian factory of medical equipments in order to understand their interactions and the impact of these interactions on organizational structure. To achieve this objective, we used a technique of analysis of social networks called blockmodels. This report describes the results of our applied study with a qualitative approach, in which was appliaed the methodology of diagnostic research. The analysis of matrixes allowed us to identify the centers and the peripheries in the networks of contacts. Among the presented diagnostics is the need for the improvement of communication processes in order to reduce problems of communication failure and to diminish the isolation of some departments.
\end{abstract}

Keywords: Social Networks; Organizational Structure; Organizacional Studies

\title{
INTRODUÇÃO
}

Redes sociais podem ser entendidas como uma configuração peculiar ao ser humano devido à sua tendência de agrupamento com seus semelhantes, levando-o a estabelecer relações dos mais variados tipos, como relações de trabalho e de amizade (Tomaél, Alcará, \& Di Chiara, 2005). Já a análise das redes sociais configura-se como uma técnica que entende os fluxos de informação e as construções sociais e simbólicas de determinados grupos, que

International Journal of Professional Business Review (JBReview), São Paulo V.2 N.1 2017, pp. 90-107, January/June 
representam um conjunto de participantes autônomos, unidos por ideias e recursos em torno de valores e interesses compartilhados (Marteleto, 2001).

Redes sociais estão presentes no ambiente corporativo, porém, a sua influência na condução dos negócios é geralmente desconsiderada como um aspecto estratégico. Neste contexto, o objetivo desta pesquisa foi analisar os contatos desenvolvidos pelos funcionários de uma organização basileira de médio porte, visando entender suas intenções e motivações, por meio da análise das redes sociais lá estabelecidas. Tal organização passou por mudança física recentemente e tem enfrentado dificuldades no estabelecimento de processos fluídos e que considerem as novas atribuições e subordinações. A partir da realização desta pesquisa, foi possível identificar a intensidade e os tipos de contatos realizados pelos funcionários e também diagnosticar a morfologia social da empresa. Ao longo da discussão dos resultados são apresentadas sugestões de intervenções possíveis que possam auxiliar a organização a estabelecer uma estrutura mais coesa, sem isolamento dos departamentos. Em termos práticos, os resultados desta pesquisa podem contribuir com organizações de médio porte com estruturas similares que enfrentam problemas causados por falha de comunicação entre departamentos. Em termos teóricos, nossa análise das redes sociais contribui com os estudos sobre estruturas organizacionais.

Além desta seção, este relato compreende outras cinco, a saber: (a) Revisão da Literatura sobre Redes Socias (b) Método adotado; (c) Resultados Obtidos e Discussão - que inclui os achados da pesquisa e as propostas de intervenção; (d) Considerações Finais e (e) Referências.

\section{REFERENCIAL TEÓRICO}

As relações entre os indivíduos vão além das estruturas e papéis formalmente estabelecidos e têm grande importância na formação da estrutura das organizações. Geralmente, as redes verbais não seguem a estrutura hierárquica da organização (Nelson \& Vasconcellos, 2007). Inclusive, muitas vezes, as informações que fluem dentro do escopo formal de hierarquias e organogramas são menos robustas e confiáveis que as informações obtidas por meio de redes de contatos (Powell, 1990).

International Journal of Professional Business Review (JBReview), São Paulo V.2 N.1 2017, pp. 90-107, January/June 
Análise das Redes Sociais de uma Fábrica de Equipamentos Médicos

Redes sociais são conjuntos de contatos que ligam vários atores (Nelson, 1984). As redes possuem três características principais: (a) conteúdo, que se refere à natureza das conexões que ligam os nós de uma rede; (b) densidade, que envolve a concentração de contatos dentro da rede e (c) configuração, a morfologia da rede (Nelson \& Vasconcellos, 2007). Estes contatos podem ser formais, informais, fortes ou fracos (Granovetter, 1973).

Para Granovetter (1973), os laços fracos fornecem mais oportunidades para que os indivíduos se integrem à comunidade, sendo fundamentais para manutenção da rede social. Laços fracos permitem ao indivíduo conectar-se com vários grupos sociais e proporcionam a formação de uma rede, sem facções isoladas entre si. Ao contrário, os laços fortes produzem uma coesão local e fragmentam uma relação. Como laços fortes são caracterizados por uma relação que possui grande intensidade de afeto, reciprocidade e frequência de contatos, as pessoas que possuem laços fortes formam ilhas de contato, o que limita sua capacidade de interação com outras redes.

Além de serem um meio importante para obtenção de informações, as redes sociais proporcionam uma forma de troca mútua de cooperação, disseminação de conhecimento, aprendizado organizacional, inovação e de compartilhamento de conhecimento (Powell, 1990). Porém, a metodologia formal de análise de redes demonstra ser pouco eficiente para realização de análise profunda das relações informais. A análise de redes sociais se apresenta, então, como uma alternativa apropriada para obter maior entendimento sobre as relações entre os indivíduos dentro de uma organização (Nelson, 1984).

\section{METODOLOGIA}

Este é um estudo aplicado, de abordagem qualitativa e o método utilizado para a sua condução foi a pesquisa diagnóstica que é uma estratégia de investigação que se propõe a explorar o ambiente, identificar e relatar os problemas, podendo até mesmo compreender propostas de solução para a organização (Martins \& Theóphilo, 2009).

Há diferentes abordagens para análise de redes sociais. A abordagem egocêntrica é mais comumente usada em estudos antropológicos. Já a abordagem gráfica é mais utilizada na

International Journal of Professional Business Review (JBReview), São Paulo V.2 N.1 2017, pp. 90-107, January/June 
sociologia. Por fim, a abordagem de blockmodeling tem sido bastante empregada em estudos de administração (Nelson, 1984). Nesta pesquisa, adotamos a abordagem de blockmodeling. Nossa opção deve-se a algumas vantagens inerentes ao seu uso. Primeiramente, nesse tipo de abordagem, os pesquisadores conseguem obter informações importantes por meio das assimetrias das interações humanas. Em segundo lugar, não é necessário entrevistar todos os participantes de uma rede, sendo possível analisar os contatos mesmo das pessoas que não participam da pesquisa (Nelson, 1984).

A abordagem de blockmodeling considera as semelhanças dentro das interações dos indivíduos e o seu objetivo é encontrar grupos de contatos que tenham o mesmo padrão. Os indivíduos que têm contatos com o mesmo padrão são agrupados em blocos. Dentre as vantagens em adotar tal abordagem está a possibilidade de obter dados importantes através das assimetrias dos blocos de contatos.

O processo de escolha da organização a ser investigada foi simbiótico, por um lado existia facilidade de acesso às informações por parte dos pesquisadores, e por outro, existia a percepção de um dos representantes da HBB Equipamentos Médicos de que uma análise das redes formadas pelos funcionários poderia fornecer explicações sobre comportamentos coletivos ou individuais não identificáveis no dia a dia.

Como primeira etapa, realizou-se a caracterização da organização, que neste estudo figura com nome fictício. Para tanto, os pesquisadores procederam a uma pesquisa exploratória baseada na análise de dados secundários coletados em documentos cedidos pela própria empresa, tais como: Histórico, Organograma e Lista de Atribuições. Os pesquisadores também analisaram dados primários, provenientes da aplicação de questionário individual e entrevista aberta com o diretor administrativo.

O questionário individual continha sete questões que versavam sobre: 1) grau de dependência para realização do trabalho; 2) grau de dependência para aprovação de projeto; 3) grau de amizade com os colegas de trabalho; 4) solicitação de conselhos pessoais; 5) solicitação de conselhos profissionais; 6) quantidade média de contatos pessoais na semana e 7) quantidade de e-mails médio enviados na semana. As questões foram cadastradas no Google Forms e os funcionários receberam um link para preenchimento online. Após recebidas as repostas, os pesquisadores digitaram todos os dados no C-NET, um aplicativo 
Análise das Redes Sociais de uma Fábrica de Equipamentos Médicos

específico para análise de redes organizacionais. Na sequencia, o software gerou uma sóciomatriz para cada questão. Por fim, foi realizada uma análise conjunta das sócio-matrizes, o que permitiu o cruzamento de dados e um maior campo de análise.

Por uma limitação temporal e de acesso, a pesquisa não pôde ser aplicada em todas as unidades da empresa HBB. Assim, o questionário foi enviado eletronicamente, pelo RH da empresa, para os funcionários alocados na unidade comercial. O universo da amostra continha 37 pessoas. Deste universo, 23 funcionários responderam, correspondendo a $62,1 \%$ da amostra. Cabe destacar que todos os diretores bem como os funcionários do financeiro e do estoque responderam ao questionário. Já o departamento de vendas foi o mais evasivo: dos 19 respondentes, $52 \%$ não responderam ao questionário.

\section{RESULTADOS OBTIDOS}

Esta seção compreende a caracterização da empresa e as análises das sócio-matrizes geradas pelo C-NET.

\section{Caracterização da empresa}

A HBB Equipamentos Médicos é uma organização de capital 100\% nacional privado que atua no Brasil e em outros países da América Latina. Suas principais atividades são a fabricação, comercialização e manutenção de equipamentos e instrumentais para vídeo laparoscopia. A HBB foi fundada em 2001, a partir da associação de duas pessoas que se conheciam por razões profissionais e em 2004, teve sua composição societária alterada, com a entrada do terceiro sócio, também profissional da área médica com larga experiência em vendas.

A empresa que começou no interior de São Paulo com cinco funcionários, hoje conta com 87 funcionários divididos em três unidades: unidade comercial em São Paulo - Capital (34 funcionários); fábrica também localizada na Capital (52 funcionários) e escritório internacional na Flórida - EUA (1 funcionário). De acordo com a classificação do Banco Nacional de Desenvolvimento Econômico e Social (BNDES) (2015), a HBB é uma média empresa, uma vez que sua receita operacional bruta está enquadrada no primeiro quartil da faixa de R\$16 a R\$ 90 milhões.

International Journal of Professional Business Review (JBReview), São Paulo V.2 N.1 2017, pp. 90-107, January/June 
Algumas alterações recentes na estrutura da empresa merecem ser relatadas, pois podem impactar na análise das redes sociais. A primeira e a mais significante delas ocorreu em dezembro de 2013, quando as áreas ligadas ao cliente final, até então alocadas na fábrica, foram transferidas para um novo prédio, situado em outro bairro. Com a mudança das instalações físicas, houve uma reestruturação do organograma. Os departamentos da assistência técnica (AT) e o de estoque de produtos acabados, até então sob responsabilidade do diretor técnico, passaram para a ser de responsabilidade do diretor administrativo.

Outra alteração foi a abertura do escritório internacional, em 2012. O objetivo de abrir um escritório fora do Brasil era o de obter certificação da FDA (Food and Drug Administration). A FDA é o órgão governamental americano que controla e regulamenta fabricantes de alimentos, medicamentos, cosméticos, equipamentos médicos e materais biológicos. O intuito de obter a certificação FDA era o de vender equipamentos para os Estados Unidos e alavancar as vendas no Brasil. Em 2014, a HBB firmou uma parceria público-privada com a Universidade Federal de Brasília, para o desenvolvimento projetos tecnológicos.

Por último, a mudança mais recente foi a instituição de um Conselho de Sócios, formado pelos três diretores e dois consultores externos. Até a finalização desta pesquisa, não havia ocorrido nenhuma reunião do Conselho.

Estudos organizacionais, em geral, tratam de abstrações que definem as características da estrutura da empresa. O tradicional organograma é a aproximação mais precisa dos relacionamentos que constituem a estrutura de uma organização, porém é limitado. Sabe-se que, na melhor das hipóteses, ele é um instrumento ambíguo e impreciso, e na pior delas, pode ser totalmente errôneo (Nelson, 1984). Geralmente, organogramas são mapas incompletos que podem esconder informações importantes, pois não descrevem todos os possíveis relacionamentos e interações dentro da organização. No caso de empresas menores, os organogramas são ainda mais imprecisos, pois em empresas menores o ambiente é mais dinâmico e a divisão do trabalho não é clara. Apesar da limitação descrita, é a partir do organograma que geralmente se comunicam as mudanças organizacionais. Além disso, é através dos organogramas que identificamos as relações de poder e as responsabilidades dos membros de uma organização (Nelson, 2006). A figura 1 ilustra o organograma da HBB.

International Journal of Professional Business Review (JBReview), São Paulo V.2 N.1 2017, pp. 90-107, January/June 


\section{=IIJPBReview}

Figura 1. Organograma da HBB Equipamentos Médicos

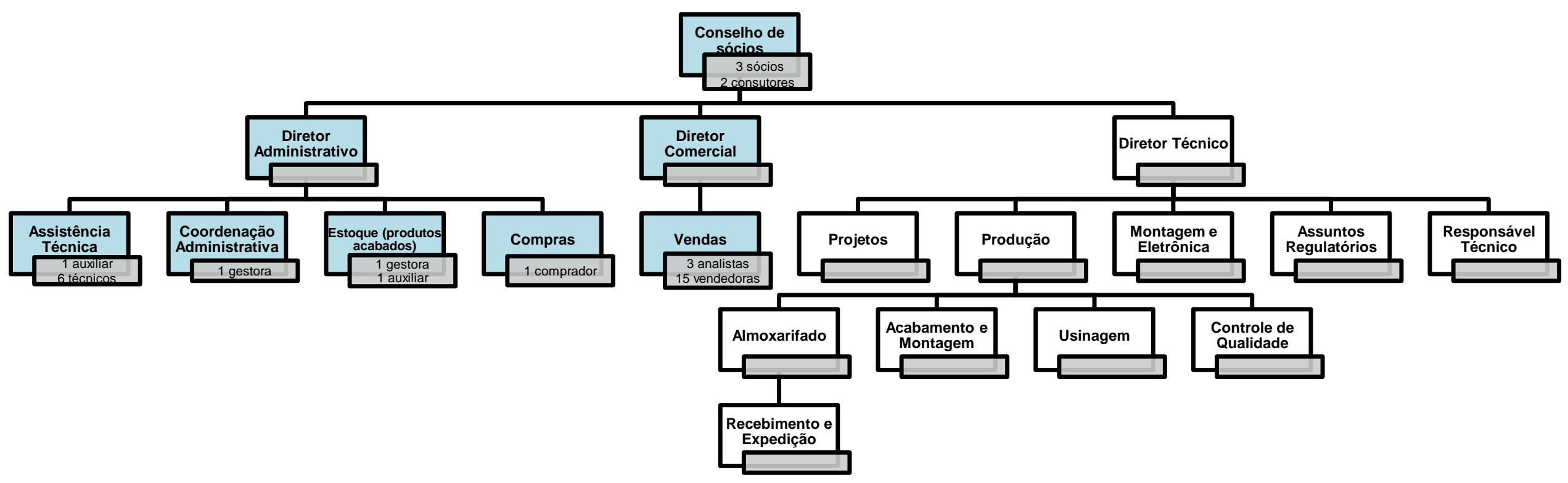

Fonte: Autores (2017) 


\section{Análise das matrizes e blockmodels}

A seguir, a análise individual de cada uma das seguintes matrizes: (a) dependência para a realização do trabalho, (b) grau de amizade, (c) contatos face a face e (d) quantidade de emails enviados. Todas geradas a partir da tabulação do questionário individual. A análises das sócio-matrizes identificou centros e periferias. Já, segunda a correlação por blockmodel permitiu comparar redes múltiplas estabelecidas pelos mesmos indivíduos e confrontá-las às estruturas formais e informais da organização, a partir da densidade de contatos (Nelson, 1984).

\section{Dependência para realização do trabalho}

Apresentamos aqui, a análise dos resultados da matriz de dependência de trabalho entre os funcionários da empresa HBB.

No centro da rede, destacam-se os três diretores da empresa (administrativo, comercial e técnico). Os resultados demonstram que no seu dia-a-dia, os funcionários dependem grandemente dos três diretores. Respectivamente, a concentração de dependência do diretor administrativo, técnico e financeiro é 109, 67 e 77. O diretor administrativo e o técnico têm uma relação de dependência equilibrada com seus subordinados. $\mathrm{O}$ primeiro tem uma concentração de dependência de outras pessoas de 103 e o segundo, 83. Por outro lado, o diretor técnico possui uma relação destoante: enquanto a concentração de pessoas que dependem dele é de 67 , ele relatou uma concentração de dependência para com outras pessoas igual a 32 .

Quanto à dependência dos funcionários da média gerência, o funcionário que obteve maior pontuação foi a coordenadora administrativa financeira com 70 pontos (pouco mais do que o diretor técnico que fica alocado na fábrica). Embora seja uma das mais demandadas ela também depende quase o dobro das pessoas para realizar seu trabalho. Uma justificativa para tal é que como "braço direito" do diretor do administrativo, esta funcionária precisa coletar informações de todas as áreas da empresa para reporta-las ao diretor. Consequentemente, ela depende diretamente de diversos funcionários para que possa concluir seu trabalho. Isto justifica o alto índice de dependência de outras pessoas (156).

Outro destaque é a coordenadora do departamento de estoque. O nível de dependência dos demais funcionários em relação a ela é de 50. Por sua vez, ela depende pouco dos seus 
colegas (21). Estes números podem indicar tal funcionária é importante na estrutura da organização e que possui bastante autonomia. Além dos três diretores e da coordenadora administrativa financeira, as pessoas com maior média de demanda são os três analistas do departamento administrativo de vendas com 63, 61 e 60.

Verifica-se que, aparentemente, o grupo de vendedores é bastante independente. Ninguém depende deles e eles dependem de poucas pessoas. A maior dependência dos vendedores é da diretoria ou do pessoal administrativo de vendas.

Interessante observar também que os técnicos dependem menos dos demais funcionários da empresa do que o contrário. Este resultado reflete o fato de que a principal atividade da organização, fabricação e manutenção de equipamentos, demanda a existência de funcionários com alto conhecimento técnico.

Algumas outras observações merecem destaque: (a) a funcionária da faxina está na formação do centro e (b) os vendedores estão geograficamente espalhados e de um total de 16 vendedores, 10 não responderam à pesquisa, possivelmente devido à baixa integração com o pessoal do escritório.

Quanto à densidade de dependência intragrupal e intergrupal para a realização do trabalho. Verifica-se que no quesito de dependência intragrupal, o maior índice ocorreu na diretoria $(2,17)$ e o menor, no departamento de vendas $(0,79)$. Quanto a dependência intergrupal, o maior índice ocorreu entre diretoria e o departamento financeiro $(3,22)$ e entre a diretoria e o estoque $(3,00)$.

\section{Grau de amizade}

Ao analisar esta questão, verificou-se que o um dos analistas do departamento de vendas (Gabriel) se considera "amigo de todos", com índice de 189 e as pessoas também se consideram suas amigas, mas não na mesma intensidade (80). Muito similar é a condição do Felipe (administrativo do AT).

Verifica-se também, que Wagner e Carlos Manuel (Vendas) se dizem amigos de todos, mas a recíproca não é verdadeira. Enquanto Wager indicou um grau de amizade de 123 com os funcionários, os funcionários relataram um grau de amizade de 36. Por sua vez, Carlos

\footnotetext{
International Journal of Professional Business Review (JBReview), São Paulo V.2 N.1 2017, pp. 90-107,
} January/June 
Manuel relatou um grau de amizade de 135, mas os funcionários indicaram que sua amizade com Carlos era de 40.

O maior índice de amizade foi declarado para o diretor administrativo, porém este diretor só tem grau de amizade 12 pelos funcionários (sendo que 8 estão concentrados na diretoria). Este possivelmente seja um viés de pesquisa. Pois este foi um questionário disparado com o aval deste diretor e os funcionários tiveram o nome identificado nas respostas.

Quanto ao grau de amizade intragrupo, a diretoria e o departamento de AT apresentaram um elevado grau de amizade entre si (5 e 3,16, respectivamente). Por outro lado, o departamento de vendas demostrou ter pouca amizade entre si $(1,22)$.

Quanto à amizade intergrupos, o departamento de vendas demonstrou ter pouca amizade com os demais departamentos da empresa. A diretoria demonstra ter amizade apenas com o departamento financeiro $(0,63)$ e de vendas $(0,16)$.

O grau de amizade da diretoria para com o grupo é bem diferente do grau de amizade do grupo para com a diretoria. Isto pode, mais uma vez, confirmar o viés da pesquisa, ou então, os diretores transparecem ser mais amigos do que de fato são. Possivelmente, não incluir o nome dos respondentes no questionário melhoraria a acurácia da pesquisa, mas inviabilizaria a análise.

\section{Quantidade de contatos face a face}

Verifica-se mais uma vez a equipe de vendas aparece na periferia da rede da empresa, pois o grupo apresenta uma baixa quantidade de contatos internos e externos. Cabe destacar que, algumas das pessoas que fazem parte do centro da rede têm bastante contato com a equipe de vendas. Estas pessoas são Gabriel (analista de vendas), Janaina (coordenadora administrativa financeira) e Felipe (administrativo da AT). É possível supor que estas pessoas têm trânsito fácil dentro do grupo de vendas. Esta densa comunicação com o grupo de vendas, que aparenta ser isolado do restante dos grupos, pode proporcionar meios de comunicação produtivos para estes três funcionários. Um dado interessante é a presença da funcionária da limpeza entre o grupo com maior número de contatos.

International Journal of Professional Business Review (JBReview), São Paulo V.2 N.1 2017, pp. 90-107, January/June 
Verifica-se que o departamento com maior densidade intra-relacional é estoque $(18,00)$, seguido pelo departamento financeiro $(17,00)$.

Ao contrário do que vimos na análise do nível de amizade e na análise do nível de dependência, a diretoria tem baixa densidade no quesito de contatos face a face $(3,17)$. Este índice não pode significar algo conclusivo, pois os diretores ficam geograficamente dispersos. O diretor administrativo e o diretor técnico estão no escritório todos os dias. Por outro lado, o diretor comercial, que reside em Sorocaba, geralmente trabalha remotamente e viaja constantemente. Devido esta configuração, os sócios se comunicam mais por telefone e WhatsApp.

\section{Quantidade de e-mails}

Nota-se que o centro de contatos é maior quando se analisa a quantidade de e-mails que são trocados entre as pessoas. Neste centro, há integrantes do departamento de vendas. Os resultados indicaram também que a maior densidade de envio de e-mails intragrupo ocorre no departamento financeiro.

\section{DISCUSSÃO DOS RESULTADOS}

Nesta seção, analisamos os resultados da análise das redes sociais da empresa HBB.

\section{Fator de dependência de trabalho}

Ao contrário do que era esperado, os resultados indicaram que os funcionários da AT dependem mais os diretor administrativo e do diretor comercial, do que do diretor técnico. Segundo o que foi relatado pelo diretor administrativo, os funcionários da AT deveriam depender mais da diretoria técnica, pois eles necessitam de orientações para o conserto dos equipamentos.

A coordenadora do departamento financeiro é a terceira funcionária mais demandada da empresa (70). Este resultado está em sintonia com o que foi coletado na entrevista com o diretor administrativo. Esta funcionária é o "braço direito" desse diretor. Segundo ele, a funcionária é considerada o pilar do faturamento, acompanha todo o processo do negócio, desde a compra de componentes até a entrega do produto para faturamento. Por isso, é correto que ela faça parte do centro da rede quanto à análise de dependência do trabalho.

\footnotetext{
International Journal of Professional Business Review (JBReview), São Paulo V.2 N.1 2017, pp. 90-107, January/June 
Possivelmente, por este motivo ela dependa de muitas pessoas para realizar o seu trabalho (pontuação de dependência de outros foi de 158).

A propósito, é importante ressaltar que o organograma da empresa não reflete esta realidade. Para ilustrar a efetiva estrutura organizacional, o organograma deveria representar coordenadora administrativa financeira por uma linha pontilhada ao lado do diretor administrativo e possivelmente ela devesse assumir um cargo que the concedesse maior poder, compatível com a responsabilidade que exerce, como o cargo de controller, por exemplo. Este fato demonstra que as redes verbais não seguem a estrutura hierárquica da organização (Nelson \& Vasconcellos, 2007; Powell, 1990). Para a manutenção dos processos do negócio, sugere-se que a HBB documente tanto quanto possível o trabalho desempenhado pela coordenadora administrativa financeira ou ainda que contratem um par para a função. Tais medidas podem no médio e longo prazo evitar o truncamento das atividades, em caso de desligamento dessa funcionária, que imprime tamanha importância na rede social da empresa.

Ao entrevistar o diretor administrativo, concluímos que será necessário pesquisar o funcionário responsável pelo departamento de compras (o departamento é composto somente por uma pessoa). Este funcionário não foi pesquisado porque ele está alocado em um prédio diferente de onde está o departamento administrativo. Há alguns meses houve uma mudança física e os funcionários administrativos e de vendas foram transferidos para outro prédio, enquanto a fábrica permaneceu na antiga instalação. Embora o departamento de compras seja subordinado ao diretor do administrativo, foi decidido manter o funcionário de compras junto com a fábrica, pois ele também é responsável pelo almoxarifado.

No âmbito de dependência do trabalho, observamos que a coordenadora do estoque tem uma posição interessante na empresa. Ela é necessária para a conclusão do trabalho de outros funcionários (pontuação 50), e ao mesmo tempo tem bastante autonomia (sua pontuação para dependência de outras pessoas é 21). $\mathrm{Na}$ verdade, ela depende somente do diretor administrativo (pontuação 4).

A estrutura da empresa é bem enxuta e horizontal, conforme demonstrado pelo organograma (Figura 1). A priori, uma estrutura como essa confere maior dinamismo e rapidez às atividades da empresa. Todavia, para confirmarmos estes dados seria necessário

International Journal of Professional Business Review (JBReview), São Paulo V.2 N.1 2017, pp. 90-107, January/June 
Análise das Redes Sociais de uma Fábrica de Equipamentos Médicos

fazer entrevista em profundidade tantos com os diretores como com funcionários da base da estrutura.

Outro dado que nos chamou a atenção é que a funcionária responsável pela limpeza está no centro da rede tanto no âmbito de dependência do trabalho como no âmbito de contatos.

\section{Fator contatos pessoais entre funcionários}

Os resultados da análise dos contatos entre funcionários confirmaram que o grupo de vendas está nas periferias da rede de contatos. Além de terem poucos contatos presenciais com os outros funcionários, os resultados indicam que os membros do departamento de vendas têm poucos contatos entre si.

Uma justificativa para este resultado é que os vendedores ficam geograficamente espalhados. Por este motivo, o meio de comunicação mais usados pelos funcionários do departamento de vendas é o telefone (o mais frequente), e-mail ou WhatsApp. Inclusive nos resultados sobre a quantidade de e-mails verificamos que este departamento envia grande quantidade de e-mails, a ponto de fazerem parte do centro da rede.

De acordo com o diretor do administrativo, os principais contatos feitos pelos funcionários de vendas são (1) com o administrativo de vendas, para solicitar e formalizar propostas; (2) com o administrativo da AT, para acompanhar processos de manutenção de equipamentos; (3) com o RH, para solicitar reserva de passagens aéreas ou de hotel; (4) com o departamento financeiro, para enviar relatório de despesas; (5) com equipe interna de vendas, para consultar status do estoque e (6) com o diretor comercial.

Cabe ressaltar, existe um grupo no Whatsapp composto pelos vendedores e diretores. Este canal de comunicação é utilizado para tratar assuntos relacionados a metas de vendas, status das vendas realizadas, promoções a serem oferecidas a clientes e comunicação interna. Há outro grupo no WhatsApp, este composto apenas pelos funcionários do departamento de vendas. $\mathrm{O}$ conteúdo das conversas deste grupo não monitorado pela diretoria.

$\mathrm{Na}$ opinião do diretor, os vendedores também se falam por telefone, pois quando um deles manda alguma comunicação indevida no grupo oficial de vendas, logo este alguém liga

International Journal of Professional Business Review (JBReview), São Paulo V.2 N.1 2017, pp. 90-107, January/June 
para o escritório e se retrata. Na opinião do diretor, os vendedores mais antigos servem de tutor aos mais novos, inclusive em questões comportamentais.

Segundo o diretor entrevistado, os vendedores são um grupo coeso. Nas duas reuniais anuais de vendas, em que participam os funcionários de vendas e os três diretores, é possível perceber que os vendedores têm bastante intimidade e amizade.

A partir destes resultados, sugerimos que a diretoria da HBB utilize a orientação informal entre os vendedores para beneficiar os negócios da organização. Uma possível alternativa é estabelecer uma tutoria informal entre os vendedores experientes e os iniciantes. Desta forma, os diretores podem canalizar a alta interação entre os vendedores para aprimorar o conhecimento e as capacidades dos funcionários.

Os vendedores Wagner e Carlos Manuel não são muito integrados ao grupo. Segundo o diretor financeiro, tais vendedores são geralmente excluídos porque nunca batem as metas de vendas. Este fato pode ser observado nos números referentes ao grau de dependência de trabalho. Enquanto Wagner e Carlos Manuel dependem muito de outros funcionários (181 e 149, respectivamente), eles não são fundamentais no trabalho dos demais - o grau de dependência dos outros funcionários em relação a eles foi baixo (22 e 29, respectivamente). Estes dados indicam que a diretoria precisa reavaliar o papel destes funcionários.

\section{Fator grau de amizade}

Verifica-se que os funcionários do departamento de AT apresentam um elevado grau de amizade (densidade 3,16) e um altíssimo grau de contato face-a-face $(10,25)$.

A disposição física deste departamento exige a divisão dos técnicos em duas salas. Thiago, Marcel e Alisson ficam em uma sala, enquanto Alessandro, Douglas, Emanuel, Felipe e Pietro ficam em outra. Esta barreira física faz com que haja certa divisão na equipe: Thiago, Marcel e Alisson não são tão unidos aos outros quatro membros do grupo. Segundo o diretor entrevistado, o grupo composto por quatro funcionários forma quase uma irmandade: são extremamente unidos a ponto de não apontarem os problemas ou limitações de seus companheiros diante da liderança, mesmo que isto impacte no desempenho da equipe.

O comportamento deste subgrupo formado por quatro funcionários (Douglas, Emanuel, Felipe e Pietro) demonstra o que Granovetter (1973) caracterizou como laços fortes, ou seja, 
nesta relação existe afeto, reciprocidade e frequência. Este tipo de comportamento acaba por confinar tais funcionários em uma rede social limitada, uma ilha dentro da HBB, o que pode comprometer o desempenho individual ou do próprio departamento. Uma possível forma de minimizar a formação destas facções seria eliminar a barreira física entre as duas salas.

Há de se destacar que o atual grau de amizade entre os diretores (5) não existia previamente, haja vista que a empresa fora constituída pela associação de três pessoas que tinham apenas laços profissionais, sem relação de parentesco ou amizade prévia. De acordo com a teoria de Granovetter (1973), a fundação da empresa poderia ser atribuída à oportunidade proporcionada por laços fracos, que permitiram aos sócios formarem uma rede proveniente de diferentes grupos sociais.

\section{CONSIDERAÇÕES FINAIS}

A análise das redes sociais da organização estudada apresentou particularidades que não estavam visíveis à diretoria. A análise dos centros e periferias e da correlação por blockmodel permitiu identificar questões extremamente relevantes da estrutura organizacional e daa morfologia da HBB, como as lacunas de comunicação, a necessidade de estruturação de processos, os riscos da alta concentração de poder e de informaçãos na coordenadora financeira e o isolamento do departamento de AT.

Como limitação da presente pesquisa destacam-se o fato de a análise contemplar organização única, pertencente a um departamento específico, o que não permite extrapolar os achados para outros setores econômicos. Outra limitação foi não aplicar o questionário com todos os funcionários da HBB. Como sugestão de pesquisa futura, recomenda-se a realização de um estudo mais abrangente, que considere todas as unidades da organização. Certamente, a análise das interações dos funcionários da fábrica, proverá dados mais conclusivos sobre o papel do diretor técnico dentro da estrutura orgaizacional. No futuro, também pretendemos realizar uma análise longitudinal da movimentação das redes sociais da unidade comercial. 


\section{REFERÊNCIAS}

Banco Nacional de Desenvolvimento Econômico e Social. (23 de Junho de 2015).

Classificação de Porte da Empresa. Fonte: http://www.bndes.gov.br:

http://www.bndes.gov.br/SiteBNDES/bndes/bndes_pt/Institucional/Apoio_Financeiro/por te.html

Biancolino, C. A., Kniess, C. T., Maccari, E. A., \& Rabechini Jr., R. (2012). Protocolo para Elaboração de Relatos de Produção Técnica. Revista Gestão e Projetos, 3(2), 294-307.

Granovetter, M. (1973). The Strength of Weak Ties (SSRN Scholarly Paper No. ID 1504479). Rochester, NY: Social Science Research Network.

Marteleto, R. M. (2001). Análise de redes sociais: aplicação nos estudos de transferência da informação. Ciência da informação, 30(1), 71-81.

Martins, G. d., \& Theóphilo, C. R. (2009). Metodologia da Investigação Científica para Ciências Sociais Aplicadas. São Paulo: Atlas.

Nelson, R. (1984). O uso da análise de redes sociais no estudo das estruturas organizacionais. Revista de Administração de Empresas, 24(4), 150-157.

Nelson, R. E., \& Vasconcellos, E. (2007). Industry Environment, National Culture, and Networks in Organizations: An Exploratory Study. Management Research, 5(3), 135-148. http://doi.org/10.2753/JMR1536-5433050301

Powell, W. W. (1990). Neither Market nor Hierarchy: Network Forms of Organization. Research in Organizational Behavior, 12, 295 - 336. http://doi.org/10.1590/S1415$\underline{65552003000200016}$

Tomaél, M. I., Alcará, A. R., \& Di Chiara, I. G. (2005). Das redes sociais à inovação. Ciência da Informação, 34(2), 93-104. http://doi.org/10.1590/S0100-19652005000200010

International Journal of Professional Business Review (JBReview), São Paulo V.2 N.1 2017, pp. 90-107, January/June 